\title{
Comparative Study on the Temporal Contrast of Femtosecond Mode-Locked Laser Oscillators
}

\author{
Nicholas Stuart, ${ }^{1,}{ }^{*}$ Timothy Robinson, ${ }^{1}$ David Hillier, ${ }^{2}$ Nick HOPPS, ${ }^{2}$ Bryn \\ Parry, ${ }^{3}$ Ian Musgrave, ${ }^{3}$ Gagik Nersisyan, ${ }^{4}$ Ahmed Sharba, ${ }^{4}$ Matthew Zepf, \\ ${ }^{4}$ ROLAND SMITH ${ }^{1}$ \\ ${ }^{1}$ Blackett Laboratory, Imperial College, London, SW7 2AZ, UK \\ ${ }^{2}$ Atomic Weapons Establishment (AWE) plc, Aldermaston, Reading, RG7 4PR, UK \\ ${ }^{3}$ Central Laser Facility, STFC Rutherford Appleton Laboratory, Harwell Oxford, Didcot, OX11 OQX, UK \\ ${ }^{4}$ Centre for Plasma Physics, Queens University, Belfast, BT7 1NN, UK \\ *Corresponding author: nicholas.stuart@imperial.ac.uk
}

Received XX Month XXXX; revised XX Month, XXXX; accepted XX Month XXXX; posted XX Month XXXX (Doc. ID XXXXX); published XX Month XXXX

We have investigated the temporal intensity contrast characteristics from a broad range of mode-locked shortpulse oscillators used for seeding high-power terawatt and petawatt class laser systems. Saturable absorber (SESAM), Kerr-lens (KLM), nonlinear polarization evolution (NPE) in optical fibers and synchronously pumped optical parametric oscillator (OPO) mode-locked sources have been measured using a third-order autocorrelator with up to $10^{10}$ dynamic range. We restricted the temporal characterization to features $<30$ ps about the laser pulse that reflect fundamental mode-locking processes. We find additional nonlinear terms and residual higher-order dispersion limits the performance of KLM and NPE sources up to the $10^{5}$ contrast level, whilst $>10^{8}$ contrast was observed from the SESAM and OPO laser pulse trains.

OCIS codes: (140.4050) Mode-locked lasers; (140.3580) Lasers, solidstate; (190.4360) Nonlinear optics, devices; (140.7090) Ultrafast lasers.

http://dx.doi.org/10.1364/OL.99.099999

The temporal intensity contrast of the short-pulse laser oscillator pulse that seeds a high-intensity laser system sets a baseline noise level for amplification to terawatt to petawatt peak-powers that are focussed onto targets at optical intensities approaching $10^{22}$ $\mathrm{W} / \mathrm{cm}^{2}$. The femtosecond to nanosecond dynamics of laser-matter experiments using these sources are crucially altered by any optical intensity noise above $\sim 10^{9}-10^{10} \mathrm{~W} / \mathrm{cm}^{2}$ that can ionize matter and form a plasma that expands away from the target before the arrival of the primary laser pulse [1,2].

In addition to a high-contrast output, sources that are suitable for seeding high-energy, high peak-power laser systems are typically $<500$ fs in duration and their emission bandwidth typically selected to be compatible with large aperture Nd:Glass amplifiers to achieve terawatt to petawatt peak-powers.

Previous investigations into the temporal contrast of short-pulse oscillators by Braun et al. compared Kerr Lens (KLM) and saturable absorber mode-locked sources using a high dynamic range secondorder autocorrelator [3]. They observed that cavities employing saturable absorbers exhibit strong suppression of ASE and other weak temporal noise features to generate $>10^{8}$ contrast pulses and that KLM sources produced considerably greater optical noise, with pedestals observed at the $10^{-5}$ intensity level.

With recent advances in laser technology, particularly with compact turn-key fibre-based ytterbium lasers [4,5] and femtosecond-pumped optical parametric oscillators (OPOs) [6,7], a broader and more detailed comparison of these laser sources is now needed. Here we present an investigation of the temporal intensity contrast across a wide-range of mode-locking schemes used in laser oscillator sources suitable, and currently used, for seeding high-power laser systems in the $1 \mu \mathrm{m}$ region, underpinning a wide range of high intensity laser matter interaction experiments. These new measurements have used a third-order autocorrelator that provides observation of asymmetric temporal processes with up to $10^{10}$ dynamic range for the $\sim 1-15 \mathrm{~nJ},<350 \mathrm{fs}, 70-80 \mathrm{MHz}$ pulse trains emitted from these sources. As far as the author is aware, this is the first time such a comprehensive comparative survey of shortpulse mode-locked oscillator contrast has been undertaken using third-order autocorrelation measurements and is also the first time the temporal contrast of fibre-based and OPO sources suitable for seeding high-energy laser systems have been measured and compared directly to saturable absorber and KLM oscillators. We have used the same diagnostic system in all of these studies to facilitate a direct comparison of their contrast performance.

To measure the temporal intensity envelope of the low-power, nanojoule, laser pulses emitted from a range of laser oscillator sources, a scanning third-order autocorrelator diagnostic (TOAD) 
was developed (Fig. 1). This was based upon the archetypal design described by Luan et al. [8]. Focused nonlinear interactions maximize the second harmonic generation (SHG) of the probe from a $1.5 \mathrm{~mm}$ thick BBO (type I) crystal and sum-frequency thirdharmonic generation (THG) signal from a 1mm long BBO (type II) sum-frequency crystal. After SHG, the fundamental and secondharmonic pulses were recollimated with an achromatic doubletand then spatially separated with a dichroic mirror. A variable delay on the subsequent fundamental wavelength beam path produces temporal scanning of the pulses. The two parallel beams enter either side of an achromatic doublet lens and focus into the sumfrequency nonlinear crystal to generate the $351 \mathrm{~nm}$ third-harmonic autocorrelation signal. A non-collinear phase-matching geometry permits spatial separation of the emerging beams and backgroundfree measurements. The third harmonic was detected with a highgain, low-noise photomultiplier (ET Enterprises 9124B) and a set of twelve calibrated density filters were used to maintain the thirdharmonic signal within the linearity range of the photomultiplier. A custom low-noise instrumentation preamplifier with $\sim 100 \mu \mathrm{s}$ integration time was used to increase the dynamic range by integrating over many hundreds of autocorrelation events of the 70-80MHz pulse trains measured.

The TOAD was found to have a temporal resolution of $\sim 200 \mathrm{fs}$, limited by the nonlinear crystal lengths, where there is a direct trade-off between linear changes in phase-matched bandwidth versus non-linear gains in conversion efficiency that are required in order to achieve up to $10^{10}$ dynamic range with $~ 10 \mathrm{~nJ}$ energy in the fundamental input pulse. Accurate measurement of the pulse duration below this limit is more routinely achieved with low dynamic range second-order autocorrelation techniques with thin crystals to permit higher resolution optical characterization.

The TOAD was aligned to each laser source with the minimum number of beam delivery optics in between to avoid introduction of

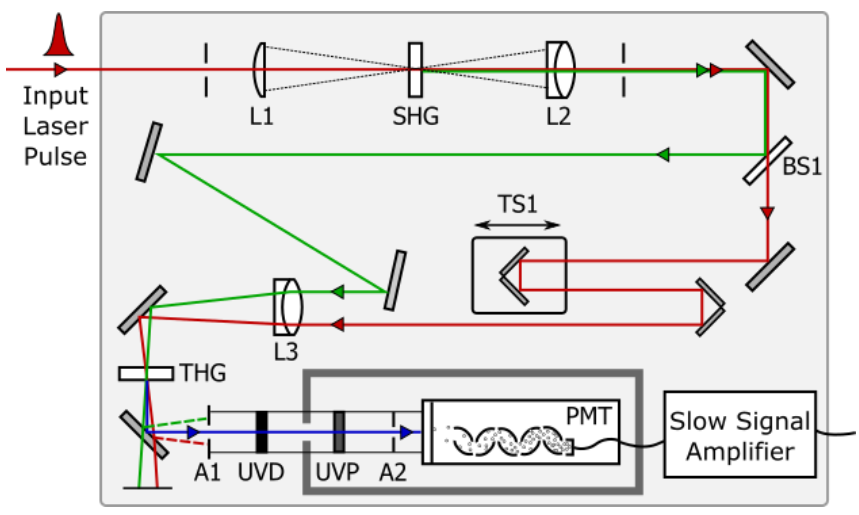

Fig. 1. Optical layout of the third-order autocorrelator diagnostic (TOAD) for high-dynamic range characterization of laser pulses. L1, L2: Focusing and recollimation optics used to maximize the intensity onto an SHG crystal, a 1.5mm thick BBO (type I). BS1: A wedged dichroic beamsplitter separates the fundamental and second harmonic pulses. TS1: Translation stage for temporal scanning. L3: An achromatic doublet lens used to focus the fundamental and second-harmonic beamlets and maximize the intensity onto the THG crystal, a $1 \mathrm{~mm}$ thick BBO (type II). A1, A2: Apertures are used to spatially filter THG light from residual fundamental and second-harmonic. UVD: Calibrated UV density filters. UVP: UV colored glass bandpass filter (UG11). secondary pulses. Each contrast trace has been fitted against a squared hyperbolic secant or Gaussian pulse to highlight deviations of the temporal intensity from these ideal cases. In comparison to discrete pre-pulses, which are device specific and can be $>10 \mathrm{ps}$ ahead of the primary pulse, we present only the close-in noise pedestal features surrounding the primary pulse, which are more indicative of the general mode-locking characteristics to be expected across all laser sources using these schemes. High contrast amplification techniques such as picosecond pumping in optical parametric chirped pulse amplification (OPCPA) will further improve the contrast against long-range noise sources $[9,10]$.

Semiconductor saturable absorber mirrors (SESAM) are widely used for mode-locking Yb-doped, Ti:Sapphire and Nd:Glass lasers. The contrast of SESAM mode-locked Nd:Glass lasers were previously found to exhibit $>10^{8}$ contrast [3]. Our contrast measurements of a SESAM mode-locked Nd:Glass laser emitting 3nJ, 310fs pulses (Time-Bandwidth GLX200HP [11]) are presented in Fig. 2. We observed $>2 \times 10^{9}$ contrast, limited by the noise floor of the TOAD diagnostic.

The fast, few hundred femtosecond, impulse response combined with modest $<1 \%$ modulation depth in the absorber effectively suppresses ASE [11,12]. The relatively slower recovery of available absorbers, typically tens of picoseconds [13], has no measureable effect on contrast. This is potentially a result of the primary pulse extracting much of the available gain in the stable mode of operation and suppressing ASE immediately behind the pulse before the population inversion can recover. Laser pulses as short as $60 \mathrm{fs}$ have been obtained with this technique [14] and 40fs pulses have been produced from Nd:Glass SESAM oscillators with use of mixed gain media [15]. Such a source, in combination with bandwidth broadening amplification from optimization of OPCPA [16] offers an attractive high-contrast seed that can potentially produce $<20 \mathrm{fs}$ equivalent amplified pulse bandwidths. The useful self-starting

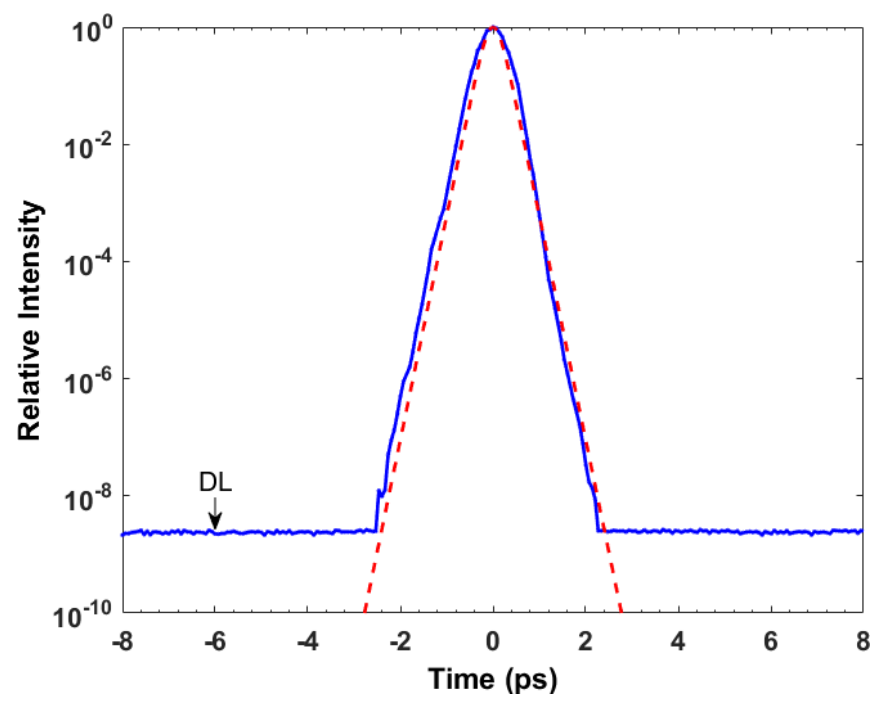

Fig. 2. Measured third-order autocorrelation of $\sim 3 \mathrm{~nJ}$ pulses emitted from a SESAM mode-locked Nd:Glass oscillator (Time-Bandwidth GLX200). (Red dashes) An ideal 400fs squared hyperbolic secant profile, which equates to a $310 \mathrm{fs}$ FWHM pulse duration after deconvolution. DL: Detection limit of the TOAD, which is $\sim 2 \times 10^{-9}$ in this measurement. 

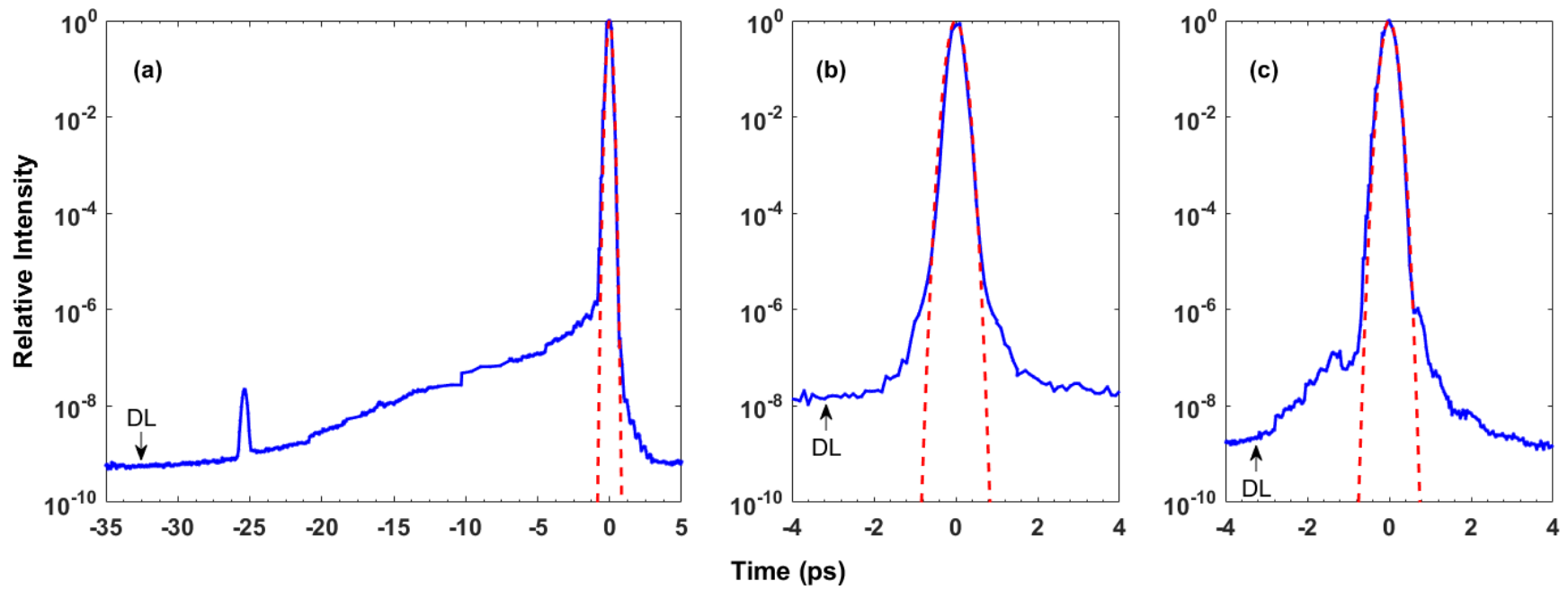

Fig. 3. Measured third-order autocorrelations of KLM laser sources. (a), (b) An AOM initiated KLM Ti:Sapphire (Spectra Physics Tsunami). (c) A KLM Ti:Sapphire (Coherent MIRA). All KLM sources were found to exhibit noise pedestals at $\sim 10^{-6}$ relative intensity. (Red dashes) All sources are fitted against a $260 \mathrm{fs}$ Gaussian intensity profile, corresponding to $~ 210 \mathrm{fs}$ FWHM duration after deconvolution, the TOAD resolution limit. DL: Detection limit of the TOAD.

properties of saturable absorbers have also been used in conjunction with Kerr-lens mode-locked cavities [17].

The optical Kerr effect is most notably harnessed for modelocking of Ti:Sapphire oscillators, frequently achieving $<20 \mathrm{fs}$ pulse durations at $800 \mathrm{~nm}$ and $<120 \mathrm{fs}$ for 1 um operation. These laser sources offer an attractive route to quickly achieving ultrafast amplified pulses. However, previous investigations by Braun et al. [3] and Jung et al. [18], using second-order autocorrelators, found short-range noise pedestals in these systems which they attributed to a combination of self-phase modulation, the temporal dependence of the Kerr effect and uncompensated third-order dispersion (TOD).

We have similarly characterized a range of KLM oscillators that lase at $1054 \mathrm{~nm}$ central wavelength and are suitable for seeding high-energy Nd:Glass amplifier systems. These sources output $\sim 120 \mathrm{fs}, 5 \mathrm{~nJ}$ pulses. Figures $3 \mathrm{a}$ and $3 \mathrm{~b}$ show the contrast performance of nominally identical commercial models of laser oscillator (Spectra Physics Tsunami) however with very different noise pedestal features that both start at $\sim 10^{-6}$ intensity level. A large asymmetric pre-pulse foot is seen in Figure 3a from one of these systems, indicative of negative net TOD or higher odd-order dispersion possibly in combination with self-phase modulation. In Figure 3c, a similar KLM Ti:Sapphire laser design (Coherent MIRA) was characterized and showed similar pedestal noise features starting between the $10^{-6}-10^{-7}$ level. Similar features have also been seen in a $100 \mathrm{fs}$ KLM Yb:KGW oscillator (Light Conversion Flint), indicating these features are most likely manifested from the KLM operation rather than being a characteristic of the Ti:Sapphire gain media but variability in the pedestal length and direction between devices are possible.

Recent technical advances have led to compact, robust modelocked fibre laser sources with $>10 \mathrm{~nJ}$ outputs using an all normal dispersion scheme and external compression [19]. However, long glass propagation lengths can lead to significant accumulation of higher-order phase terms, particularly positive third-order phase. Additionally, as NPE depends upon the optical Kerr effect to produce elliptical rotation, strong self-phase modulation effects are likely to occur, producing spectral modulations which may then translate to temporal modulations after dispersion.

We have characterized the contrast of a short-pulse Yb-doped fibre laser producing $50 \mathrm{fs}$ pulses that are externally compressed with a prism pair (Coherent Fidelity 2). The results in Figure 4 show the strong presence of a post-pulse foot starting at $\sim 10^{-5}$ relative intensity with a decaying oscillatory modulation indicative of positive higher odd-order dispersion, also likely in combination with self-phase modulation. We observed $>3 \times 10^{8}$ pre-pulse contrast, limited by the noise floor of the TOAD diagnostic. However, nonlinear mechanisms that act to convert the large postpulse noise intensity into pre-pulse noise will need to be controlled to maintain this low level of pre-pulse [20].

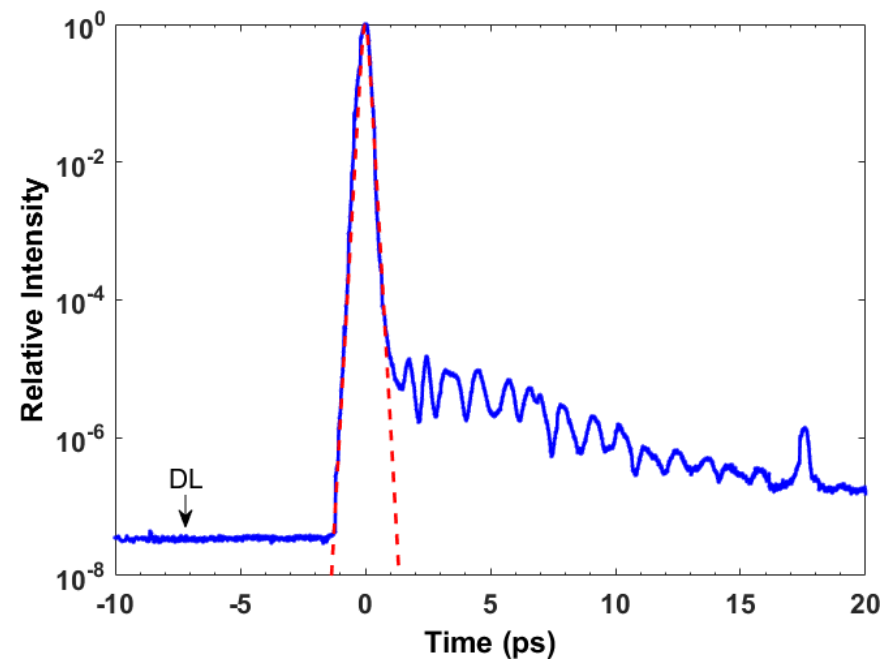

Fig. 4. Measured third-order autocorrelation of the NPE mode-locked Yb-doped fiber laser (Coherent Fidelity 2). (Red dashes) The $~ 210 \mathrm{fs}$ Gaussian FWHMTOAD resolution limit. DL: Detection limit of the TOAD, which is $\sim 3 \times 10^{-8}$ in this measurement. 
Large advances have also been made in the area of optical parametric oscillators (OPOs). These are external cavities with a broadly phase-matched difference frequency nonlinear crystal pumped by a mode-locked laser. The signal and idler pulse durations are closely linked to the pump duration driving the nonlinear amplification. The use of short crystal lengths and allreflective designs with minimal dispersion control can achieve $20 \mathrm{fs}$ pulse durations with very broad $(1-5 \mu \mathrm{m})$ wavelength tunability when pumped with ultrafast Ti:Sapphire lasers [6].

We present the contrast characterization of a multi-watt $\mathrm{Yb}$ Fiber laser pumped OPO that produces 12nJ, 120fs pulses at $1054 \mathrm{~nm}$ wavelength (Spectra Physics Insight DS+ [21]). The TOAD was able to measure the pulse temporal intensities with $2 \times 10^{10}$ dynamic range.

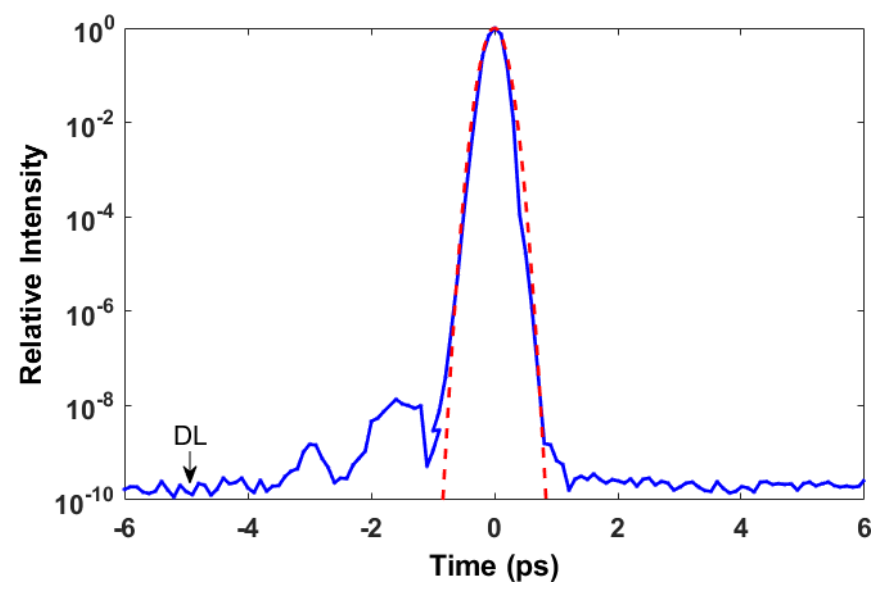

Fig. 5. Measured third-order autocorrelation of a Yb-Fiber laser pumped optical parametric oscillator (Spectra Physics Insight DS+). (Red dashes) The $\sim 210$ fs Gaussian FWHM TOAD resolution limit. DL: Detection limit of the TOAD, which is $\sim 2 \times 10^{-10}$ in this measurement.

Short-lived pre-pulsefeatures at $-1.5 \mathrm{ps}, 10^{-8}$ relative intensity and $-3 \mathrm{ps}, 10^{-9}$ relative intensity were observed, which are likely either residual negative odd-order phase features or discrete pre-pulses generated by thin optics in the cavity. The contrast performance of the OPO significantly improves upon that of the KLM and NPE lasers, as self-phase modulation is not phase-matched and shortgain media with ultrafast pumping reduces accumulation of higherorder phase. The wide tunability of an OPO, high output powers and comparable <150fs pulse durations, in addition to the now confirmed high-contrast output, offers an attractive option for seeding advanced high-power lasers.

In summary, we have experimentally characterized the temporal intensity contrast of SESAM, KLM, NPE and OPO mode-locked laser oscillators that are suitable for seeding amplifier chains that operate in the $1 \mu \mathrm{m}$ region with a high-dynamic range third-order autocorrelator. We found SESAM mode-locked laser sources produced the most ideal pulses, with no noise pedestal detected within the $2 \times 10^{9}$ dynamic range of the measurements, limited by the optical intensities from these sources without additional amplification. Suspected unwanted nonlinearities and/or higherorder phase produce symmetric and asymmetric noise features at $\sim 10^{-5}$ and $10^{-6}$ relative intensity in NPE and KLM lasers respectively, which both use the optical Kerr effect as their primary modelocking mechanism. This level of optical noise may become contrast limiting sources for short-pulse, high-power, high-contrast laser systems. Potentially manageable pre-pulse at the $10^{-8}$ level was observed from an OPO source, which also offers large wavelength tunability in comparison to other mode-locked sources.

Funding. Engineering and Physical Sciences Research Council (EPSRC) (EP/G001324/1); AWE Industrial CASE Studentship.

Acknowledgements. We are grateful to Amelle Zair and Konstantin Holzner for providing access to a Yb:KGW KLM laser source (Light Conversion Flint).

\section{References}

1. P. McKenna, F. Lindau, O. Lundh, D. Neely, A. Persson, and C.-G. Wahlström, Philos. Trans. R. Soc. Lond. Math. Phys. Eng. Sci. 364, 711 (2006).

2. G.M. Petrov, C. McGuffey, A.G.R. Thomas, K. Krushelnick, F.N. Beg, J. Appl. Phys. 119, 053302 (2016).

3. A. Braun, D. Kopf, I. D. Jung, J. V. Rudd, H. Cheng, K. J. Weingarten, U. Keller, and G. Mourou, Opt. Lett. 20, 1889 (1995).

4. F. Ilday, J. Buckley, L. Kuznetsova, and F. Wise, Opt. Express 11, 3550 (2003).

5. X. Zhou, D. Yoshitomi, Y. Kobayashi, and K. Torizuka, Opt. Express 16, 7055 (2008).

6. S. Chaitanya Kumar, A. Esteban-Martin, T. Ideguchi, M. Yan, S. Holzner, T. W. Hänsch, N. Picqué, and M. Ebrahim-Zadeh, Laser Photonics Rev. 8, L86 (2014).

7. O. Kokabee, A. Esteban-Martin, and M. Ebrahim-Zadeh, Opt. Lett. 35, 3210 (2010).

8. S. Luan, M. Hutchinson, R. Smith, and F. Zhou, Meas. Sci. Technol. 4, 1426 (1993).

9. I. Musgrave, W. Shaikh, M. Galimberti, A. Boyle, C. Hernandez-Gomez, K. Lancaster, and R. Heathcote, Appl. Opt. 49, 6558 (2010).

10. D.I. Hillier, S. Elsmere, M. Girling, N. Hopps, D. Hussey, S. Parker, P. Treadwell, D. Winter, T. Bett, Appl. Opt. 53, 6938 (2014).

11. D. Kopf, K. J. Weingarten, F. X. Kärtner, and U. Keller, Opt. Lett. 20, 1169 (1995).

12. L. R. Brovelli, U. Keller, and T. H. Chiu, J. Opt. Soc. Am. B 12, 311 (1995).

13. U. Keller, K. J. Weingarten, F. X. Kartner, D. Kopf, B. Braun, I. D. Jung, R. Fluck, C. Honninger, N. Matuschek, and J. Aus der Au, IEEE J. Sel. Top. Quantum Electron. 2, 435 (1996).

14. J. Aus der Au, D. Kopf, F. Morier-Genoud, M. Moser, and U. Keller, Opt. Lett. 22, 307 (1997).

15. S. Han, W. Lu, B. Y. Sheh, L. Yan, M. Wraback, H. Shen, J. Pamulapati, and P. G. Newman, Appl. Phys. B 74, s177 (2014).

16. D. Bigourd, S. Patankar, S. I. O. Robbie, H. W. Doyle, K. Mecseki, N. Stuart, K. Hadjicosti, N. Leblanc, G. H. C. New, and R. A. Smith, Appl. Phys. B 113, 627 (2013).

17. R. Fluck, I. D. Jung, G. Zhang, F. X. Kärtner, and U. Keller, Opt. Lett. 21, 743 (1996).

18. I. D. Jung, F. X. Kärtner, J. Henkmann, G. Zhang, and U. Keller, Appl. Phys. B 65, 307 (1997).

19. J. R. Buckley, F. W. Wise, F. ö. Ilday, and T. Sosnowski, Opt. Lett. 30, 1888 (2005)

20. N.V. Didenko, A.V. Konyashchenko, A.P. Lutsenko, S.Y. Tenyakov, Opt. Express. 16, 3178 (2008)

21. J. D. Kafka, C.-Y. Chien, Y. Deng, A. C. Florean, D. E. Spence, and J. Zhou, U.S. patent US20110180729 A1 (2011). 